\title{
Design Science Research Contribution to Business Intelligence in the Cloud - A Systematic Literature Review
}

\author{
Odette Sangupamba Mwilu ${ }^{1}$, Isabelle Comyn-Wattiau ${ }^{1,2}$ and Nicolas Prat ${ }^{2}$ \\ ${ }^{1}$ CEDRIC-CNAM, Paris, France \\ odemwilu@hotmail.com, isabelle.wattiau@cnam.fr \\ ${ }^{2}$ ESSEC Business School, Cergy-Pontoise, France \\ $\{$ wattiau,prat\}@essec.edu
}

\begin{abstract}
Business intelligence (BI) helps managers make informed decisions. In the age of big data, BI technology provides essential support for decision making. Cloud computing also attracts many organizations because of its potential: ubiquitous, convenient, on-demand network access to a shared pool of configurable computing resources (e.g. networks, servers, storage, applications, and services). This paper focuses on the deployment of BI in the cloud, from the vantage point of design science research (DSR). We produce a state of the art of research pertaining to BI in the cloud, following the methodology of systematic literature review. This literature review especially exhibits the different artifacts proposed by design science researchers regarding BI in the cloud. To structure the literature review, we propose a framework composed of two dimensions: artifact type and BI step. In particular, we propose a typology of artifact types, refining the coarse-grained typology commonly used in DSR. We use the two-dimensional framework both to map the current state of DSR regarding BI in the cloud, and to elicit future research avenues in terms of design science artifacts for BI in the cloud. The contribution is threefold: the literature review may help DSR researchers get an overview of this active research domain; the two-dimensional framework facilitates the understanding of different research streams; finally, the proposed future topics may guide researchers in identifying promising research avenues.
\end{abstract}

Keywords: business intelligence, analytics, cloud computing, design science research, systematic literature review

\section{Introduction}

Business intelligence (BI) helps managers make informed decisions. In the age of big data, the support of BI technology is critical in guaranteeing effective and efficient decision making. BI tools facilitate the presentation of more accurate reporting, improve decision making, enhance customer relationships, and increase revenue [1]. BI must scale up to big volumes of data (big data analytics). The term of business analytics appeared in 2008 and tends to replace business intelligence. According to IDC [3], the business analytics software market will grow at a 9.7\% compound annual rate through 2017. Even if the BI software market is mature and prolific, many research issues remain open in this domain. BI is a major topic since companies need to acquire more skills and to increase their maturity. They need robust methodologies to choose BI solutions, to implement them, to express business goals in terms of indicators and, more generally, to manage companies thanks to effective decision support systems.

Cloud computing also attracts many organizations because of its potential: ubiquitous, convenient, ondemand network access to a shared pool of configurable computing resources (e.g., networks, servers, storage, applications, and services) [2]. It provides innovative services to different types of users. The latter are freed from the underlying technical infrastructure. Beyond outsourcing, two concepts are highlighted in cloud computing: virtualization and agility. Through the cloud, organizations can acquire IT services without human intervention from the provider. According to IDC [3], spending on public IT cloud services alone was estimated a $\$ 47.4$ billion industry in 2013 and is expected to more than double by 2017. Ultimately, cloud computing enables more efficient BI tasks. It allows faster deployment and greater flexibility compared to traditional BI solutions [4] and produces accurate results more rapidly than desktop computers [5]. Even if the cloud relies on well-known technologies, it raises new research questions: which guidelines are available for companies that want to adopt cloud architectures? Which architecture must be preferred, given a context? 
Are there specific approaches to help a company migrate to the cloud? Hence, the many issues and opportunities associated with cloud computing also generate a dynamic research activity.

$\mathrm{BI}$ and analytics and cloud computing raise many issues for information systems researchers from various streams, more specifically quantitative research, qualitative research, and design science research. In this paper, we take the vantage point of design science research (DSR). DSR builds and evaluates artifacts [6], which may be constructs, models, methods, or instantiations [7]. DSR has much to contribute to BI and analytics [8] and cloud computing [9]. We consider the intersection of these two topics, focusing on the deployment of BI in the cloud, from the point of view of DSR. Our research question is: What new artifacts can design science researchers bring to the domain of BI in the cloud? To answer this question, we perform a systematic literature review (SLR) of BI in the cloud in DSR. The literature review exhibits the different artifacts proposed by design science researchers regarding BI in the cloud. Moreover, building on the literature and on a framework composed of two dimensions (artifact type and BI step), we elicit future research avenues in terms of design science artifacts for BI in the cloud. This article is an extension of a previous paper [2]: it proposes a systematic literature review (SLR) and enriches the two-dimensional framework.

The remainder of the paper is structured as follows: in the ensuing section, we present the research gap and the research question. In Section 3, we describe our research method. Section 4 details our typology of DSR artifacts. In Section 5, we propose a framework synthesizing the current state of research for business intelligence in the cloud. Section 6 describes open research issues and opportunities for DSR and introduces a discussion before concluding the paper in Section 7.

\section{Research gap and research question}

Design science produces artifacts to attain human goals [10]. DSR in information systems (IS) seeks to extend the boundaries of human and organizational capabilities by creating new and innovative artifacts [6]. While behavioral research aims at understanding, the main objective of DSR is utility. DSR is now established as a major research paradigm in IS [11]. Many researchers support the view that the output of DSR is an artifact or a set of artifacts, even though others argue that its purpose should be the creation of theories [12]. In this paper, we adopt the view that the primary goal of DSR is the production of useful artifacts. March and Smith [7] distinguish four categories of artifacts: constructs, models, methods, and instantiations. According to these authors, constructs "form the vocabulary of a domain. They constitute a conceptualization used to describe problems within the domain and to specify their solutions". A model "is a set of propositions or statements expressing relationships among constructs". A method is "a set of steps (an algorithm or guideline) used to perform a task. Methods are based on a set of underlying constructs (language) and a representation (model) of the solution space". An instantiation is "the realization of an artifact in its environment". This typology of artifacts is the most widely used in DSR. Other typologies have been proposed, e.g. that of Offermann et al. [13]. These typologies help researchers and practitioners represent, analyze, design, implement, and evaluate successful information systems. Our aim is to study which artifacts have been proposed regarding BI in the cloud. Building on these previous papers, we proposed a typology of artifacts that allows us to structure the literature review on BI in the cloud and detect open research questions [2].

Many artifacts have been designed and found valuable regarding BI. Cloud computing puts forward new architectures and new opportunities to share information and applications, but it also raises new risks. It may offer new economic models for information technology (IT) and IS solutions. BI and analytics rely on huge data sets, requiring complex and robust algorithms to produce information and knowledge. Abundant literature can be found on BI and cloud computing and, more recently, on their combination.

The recent literature describes many research works investigating the specific opportunities and addressing the research issues of BI in the cloud. Since this is a recent topic, there is no comprehensive survey allowing researchers to quickly obtain a synthesized view of research results and opportunities. This paper aims at filling this gap by proposing a systematic literature review and a framework for analyzing significant results and identifying opportunities for further research. Considering the significant contribution of DSR to BI in the cloud, the paper focuses on this research paradigm.

$\mathrm{BI}$ in the cloud raises many new research issues for the IS community at large (quantitative and qualitative research, IS economics, design-science research). We acknowledge the potential contributions of the various research paradigms to BI in the cloud, especially when used in combination (e.g. combination of 
DSR with qualitative research), but we focus on DSR to reduce the search space. Thus, the research question addressed in the paper is: What new artifacts can DSR bring to the domain of BI in the cloud?

In a previous publication [2], we proposed a first answer to this question. We conducted a preliminary literature review to evaluate the state of the art of DSR for BI in the cloud. Using our artifact typology, we summarized this review by listing, for each artifact type, which functions were covered among data management, service management, and security management. More precisely, for each artifact type and for each function, we answered the following question: did authors propose such artifacts to manage this function (data, service, security) of BI in the cloud? Then, based on this preliminary literature review, we proposed future research avenues for $\mathrm{BI}$ in the cloud.

In this paper, we go beyond by first performing a systematic literature review to validate and strengthen our findings. Second, we organize the results into a two-dimensional framework. The first dimension is our typology of artifacts. The second one is dedicated to BI components. In the same way, we both analyze the literature and suggest further research according to this framework. The next section describes our research method.

\section{Research method}

In this paper, we present the result of a systematic literature review (SLR) that we performed to assess the current state of research on $\mathrm{BI}$ in the cloud.

Kitchenham [15] mentions three common motivations for undertaking a SLR in software engineering: 1) to summarize the existing evidence, the benefits and limitations of a specific technology, 2) to identify any gaps in current research and suggest areas for further investigation, and 3) to provide a framework in order to appropriately position new research activities. This paper is in alignment with the last two motivations, focusing on BI in the cloud from the vantage point of DSR.

Let us note that a SLR synthesizes existing work in a scientific approach, targeting the completeness of the search to be assessed. In particular, it allows literature reviews not to be limited to academic publications. This seems particularly useful in emergent topics, such as BI on the cloud, where both researchers and professionals may propose new artifacts.

\subsection{Research question}

Let us remind our research question identified in the previous section: Which new artifacts can DSR bring to the domain of BI in the cloud?

According to the principles of SLR, we first have to check that the research question is meaningful and important to practitioners as well as researchers. By describing the market of BI solutions and the advantages of the cloud, we have met the practitioners' viewpoint. BI and cloud computing being two major research topics in computer science and IS, the interest of combining both concepts is at least twofold: BI in companies becomes a more and more important part of the information system, requiring investment in terms of computational performance and data volumes, that the cloud may provide easily; second, given the cloud opportunities, the question of migrating the whole IS including its BI is of great interest. In particular, researchers have to provide professionals with models and methods helping to make the decision as well as to implement it.

The second requirement is that answering the research question will impact current practice and extend the body of knowledge. Practitioners need to know if past rules for managing BI remain valid if it is based partially or totally on the cloud. Research must check the applicability of past artifacts and when appropriate propose new ones.

Third, the answer to the research question must identify discrepancies between common beliefs and reality. In our case, the question appears to be: is cloud computing a new concept or only a new deployment of past concepts (e.g. distributed systems)? Regarding BI, the question turns into: does migration of BI on the cloud lead to new challenges and thus require new artifacts such as methods for example?

\subsection{Selection of papers}

Our sample for SLR first included articles that are at the intersection of BI and cloud computing on the one hand (sources of type 1). In order to select the relevant references [15], we used appropriate keywords for each type of sources. The following set of keywords was used: (« Business Intelligence » OR « Analytics » OR « Data warehouse ») AND («Cloud $» \mathrm{OR} « \mathrm{SaaS} »)$. The first set of terms refers to BI and its 
synonyms, and the second refers to the cloud and its most typical layer (SaaS). We searched the keywords in the full text of papers.

Apart from Google Scholar, the main sources of academic papers related to BI in the cloud are the ACM Digital Library, IEEE Xplore, DBLP, ScienceDirect, EBSCOhost and the electronic library of the AIS (AISeL). We used all these sources. One main objective of SLR is the completeness of results, constraining us to browse all common academic sources.

Based on initial tests, we limited the search to the first fifty pages of results, for each source. The time span for sources examination was from 2004 to 2014, considering that BI and cloud computing technologies are evolving fast. Several papers were cited in multiple sources (in particular, most papers referenced in Google Scholar). Thus, the different search results were then merged (deduplication).

We examined these papers to exhibit artifacts already proposed in the papers, and also artifacts mentioned as future research.

On the other hand, given that BI in the cloud is an emerging technology, we took into account papers that deal with each of these two domains separately (sources of type 2). The process for selecting these papers was similar to the one described above for papers on the topic of BI in the cloud. The difference lies in the choice of keywords: the keywords were specific to each of the related domains, and also ensured that only the seminal papers for understanding the domains (literature reviews) were selected. We queried the same databases with two consecutive queries as follows:

- ("Overview" OR "State of the art" OR "Survey" OR "Literature review”) AND ("Business

Intelligence" OR "Analytics" OR "Data warehouse")

- ("Overview" OR "State of the art" OR "Survey" OR "Literature review”) AND ("Cloud" OR "SaaS”).

The objective was not to analyze the whole literature on each topic but to obtain a content synthesis of each domain. Thus, we limited our SLR to the papers proposing such a synthesis. After deduplication and elimination of non-ERA and non-DSR papers, the list was reduced to 19 papers and we examined these papers to exhibit artifacts already proposed in the papers.

\subsection{Paper quality assessment}

Conducting an SLR requires the definition of exclusion and inclusion criteria ensuring the quality of selected papers and reducing the bias in this selection.

As an exclusion criterion of our SLR, we eliminated all the papers that were not published in journals or conference proceedings from the ERA list [16] [17] (exclusion criterion). Then the abstracts were screened to ensure that they were DSR papers (inclusion criterion). The basic heuristic helping us decide if a paper was a DSR paper was to elicit the artifact or the artifacts proposed in the paper (inclusion criterion). Thus, our typology of artifacts (presented below) allowed us to detect and categorize the artifacts already proposed in the domain of BI in the cloud, and hence those to which DSR should dedicate its future efforts.

Originally, after querying the databases mentioned above, we got a list of 1030 papers. After deduplication and elimination of non-ERA and non-DSR papers, the list was reduced to 63 papers (45 of source 1 and 18 of source 2 ).

\subsection{A two-dimensional framework for characterizing DSR pertaining to BI in the cloud}

In this section, we describe the two dimensions of our literature analysis, i.e. the artifacts described in papers and the steps of business intelligence. These dimensions aim to help the reader in understanding the field. Below, we describe and justify these two dimensions.

We based our categorization on the hierarchy of artifacts detailed in Section 4 below. This typology helped us in different ways. First, we focused our SLR on DSR. This typology of artifacts proved very useful in deciding whether a paper was a contribution of DSR. When parsing more than one thousand abstracts, we detected whether they mentioned as a contribution one or several artifacts of our typology. Then we read the whole paper to ensure that we had captured the whole set of DSR artifacts proposed by the authors.

Business intelligence requires the capabilities of acquiring information, storing it, and analyzing it using different tools and techniques. These three aspects are often handled through different research teams. We structured our state of the art along these three aspects: 
- Data collection and blending: This aspect includes ETL (Extract-Transform-Load) techniques for collecting and consolidating data from different sources.

- Data modeling and storage: This topic encompasses all the models, methods, techniques, and tools for managing high volumes of various data types.

- Analytics: This third aspect encompasses the analysis and visualization of data. It may be subdivided into descriptive, inquisitive, predictive, and prescriptive analytics [18] [19].

Given the definition of BI that involves collecting, blending, modeling, storing, analyzing and reporting information, this categorization is used to classify the artifacts according to their role in this process.

In the following section, we describe our typology of design science research artifacts as a help for structuring our analysis.

\section{A detailed typology of DSR artifacts}

DSR in IS generates new and innovative artifacts [6]. As mentioned above, March and Smith [7] distinguish four types of artifacts constituting the outputs of DSR: constructs, models, methods, and instantiations. This typology of artifacts is widely used, including in the seminal paper by Hevner et al. [6]. However, the typology is sometimes difficult to operationalize, due to the relative fuzziness of the concepts of construct, model, method, and instantiation. Therefore, it is useful to specialize the typology, specifying and defining subcategories for the four main categories of artifacts. As an example, Offermann et al. [8] specialize the typology of artifacts. This work provides a useful basis for a classification of the various types of artifacts. However, the typology of Offermann lacks some important subcategories of artifacts, some types include a large number of different concepts, and the proposed definitions may lead to confusion. Moreover, the proposed types are not directly related to the original concepts of constructs, models, methods, and instantiations. Consequently, we proposed our typology of the different types of artifacts, subtyping the categories of construct, model, method, and instantiation [2]. For each subcategory, we gave a definition, using or adapting the definitions found in the literature. Our typology, with precise subcategories and a definition for each subcategory, helps in the identification and characterization of DSR artifacts. When the definition is borrowed from other authors, the reference is provided.

\subsection{Constructs}

We have put together, in the construct category, the concepts and their combinations, leading to three artifacts: concepts, language, and meta-model.

- Language: A set of concepts, or more generally symbols, rules for combining them (syntax), and rules for interpreting combinations of symbols (semantics) [20].

- Meta-model: A set of concepts represented in graphical notation, with rules for combining the concepts.

- Concept: A new construct added to an extant language or meta-model. As an example, class is a main concept in object-oriented models as well as verb is a main concept in natural language grammars.

\subsection{Models}

When constructs are used to build more structured objects, we obtain models. The most common artifact in the model category is the system design [13], proposed in a large number of DSR papers. We have added the categories of ontologies, taxonomies, and frameworks, that are very specific models frequently proposed in DSR.

- System design: A structure or behavior-related description of a system, commonly using some graphical notation and possibly text [13].

- Ontology: An explicit formal specification of a shared conceptualization [21]

- Taxonomy: A classification of objects in a domain of interest, based on common characteristics [22].

- Framework: A logical structure for organizing complex information [23].

- Architecture: A blueprint representing the fundamental organization of a system embodied in its components, their relationships to each other, and to the environment [24] [25].

- Requirement: A condition or capability that must be met or possessed by a system [24]. 


\subsection{Methods}

The method category puts together dynamic artifacts. Offerman et al. [13] proposed: method, guideline, and algorithm. We added method fragment for describing components of more comprehensive methods, including patterns for example. We also added metrics. The latter are close to algorithms but very specific artifacts since their interest is not their logic or their performance but their ability to synthesize a measure inside a mathematical formula. Finally, we adopted methodology instead of method, thus avoiding homonyms between categories and subcategories.

- Methodology: A predefined set of steps and guidelines, with associated techniques and tools. It is aimed at, or used by, individuals who work in a discipline [24] [26].

- Guideline: A suggestion regarding behavior in a particular situation [13]. Examples: design principles (broad guidelines), heuristics, rules (detailed guidelines) [27].

- Algorithm: An executable sequence of operations for performing a specific task [24].

- Method fragment: A method component that can be treated as a separate unit and reused in different contexts [28]. Design patterns are an example of method fragment.

- Metric: A function that assigns a number or symbol to an entity in order to characterize an attribute or a group of attributes. The value of the metric is called a measure [29].

\subsection{Instantiations}

Instantiations are specific artifacts often proposed to assess the feasibility of other constructs, e.g. system designs or methodologies. We differentiate between the prototypes or more finalized tools on the one hand and, on the other hand, the examples described in the papers to illustrate the contributions.

- Implemented system: An implemented software or hardware system. In research papers, the implemented systems may be either prototypes or finalized tools.

- Example: Any other concrete materialization of an abstract artifact (construct, model, or method). Examples are proposed either for illustrating the artifact or as a first step of validation of the usefulness of the artifact. It may be the application of a query language to an illustrative scenario, or the illustration of a design-theory framework with concrete examples of design theories, or the application of a project methodology to a real project.

We applied our typology to a set of more than 120 papers and thus checked that it is a useful tool to check if a paper is dedicated to DSR and to extract the main contributions of such papers [30].

\section{Mapping current research on $B I$ in the cloud}

In this section, we describe the findings from our SLR. Table 1 synthesizes, for each BI step, the artifacts found in the 64 papers. It illustrates the richness of DSR research on the topic. Almost each cell of the table contains many different artifacts. 


\begin{tabular}{|c|c|c|c|c|}
\hline Artifact & $\begin{array}{c}\text { Data } \\
\text { collection and } \\
\text { blending }\end{array}$ & $\begin{array}{l}\text { Data modeling } \\
\text { and storage }\end{array}$ & Analytics & $\mathrm{BI}$ as a whole \\
\hline Language & & $\begin{array}{l}\text { - Medical markup } \\
\text { language [47] }\end{array}$ & & \\
\hline Concept & & & $\begin{array}{l}\text { - BIaaS concept [59] } \\
\text { - CaaaS concept [60] }\end{array}$ & \\
\hline $\begin{array}{l}\text { System } \\
\text { design }\end{array}$ & & $\begin{array}{l}\text { - Data structure in the } \\
\text { cloud [49] }\end{array}$ & $\begin{array}{l}\text { - Business process for } \\
\text { incorporating BI in cloud market } \\
\text { [78] } \\
\text { - Business process for a generic } \\
\text { value network [77] }\end{array}$ & \\
\hline Taxonomy & & & $\begin{array}{l}\text { Taxonomy of main aspects for } \\
\text { cloud monitoring [79] } \\
\text { - Taxonomy of data mining } \\
\text { algorithms [69] }\end{array}$ & $\begin{array}{l}\text { - Taxonomy of } \\
\text { mobile cloud } \\
\text { computing [31] }\end{array}$ \\
\hline Framework & $\begin{array}{l}\text { Framework for } \\
\text { data integration }\end{array}$ & $\begin{array}{l}\text { Framework for } \\
\text { hypercube }\end{array}$ & $\begin{array}{l}\text { - Framework for BI\&A technology } \\
\text { [76] }\end{array}$ & $\begin{array}{l}\text { - Framework of cloud } \\
\text { BI [33] }\end{array}$ \\
\hline & $\begin{array}{l}\text { - G11] } \\
\text { Generic } \\
\text { framework for } \\
\text { interactive } \\
\text { clouds [42] }\end{array}$ & generation [46] & $\begin{array}{l}\text { - Framework for provisioning } \\
\text { resources from a public cloud } \\
\text { [74] } \\
\text { - Framework for healthcare } \\
\text { delivery [75] } \\
\text { - OLAP framework [63] [64] } \\
\text { - Data mining cloud framework's } \\
\text { graphical interface [70] } \\
\text { - Framework for BI research [81] } \\
\text { - Framework for cloud data } \\
\text { analytic [65] } \\
\text { - Framework to verify security in } \\
\text { the cloud [82] }\end{array}$ & $\begin{array}{l}\text { - Framework of } \\
\text { social BI research } \\
\text { agenda [39] } \\
\text { - Framework of cloud } \\
\text { BI services [34] }\end{array}$ \\
\hline $\begin{array}{l}\text { Architectur } \\
\text { e }\end{array}$ & & $\begin{array}{l}\text { - Cloud computing } \\
\text { architecture [48] } \\
\text { - Cloud data } \\
\text { warehouse } \\
\text { architecture [49] } \\
\text { - Objective-driven } \\
\text { architecture [50] } \\
\text { - Elastic data storage } \\
\text { architecture [51] } \\
\text { - Architecture for } \\
\text { building OLAP } \\
\text { cubes in the cloud } \\
\text { [52] } \\
\text { - Architecture of a } \\
\text { system for data } \\
\text { analytics in the } \\
\text { cloud [53] }\end{array}$ & $\begin{array}{l}\text { - Architecture for online } \\
\text { aggregation in the cloud [61] } \\
\text { - Architecture of analytics on the } \\
\text { cloud [67] } \\
\text { - DSS Architecture [80] } \\
\text { - Architecture to maintain the } \\
\text { elastic OLAP cloud platform [62] } \\
\text { - Architecture of a security system } \\
\text { [83] } \\
\text { - Architecture for scalable runtime } \\
\text { optimized of cloud data analytics } \\
\text { [78] }\end{array}$ & $\begin{array}{l}\text { - Architecture of } \\
\text { cloud BI [35] } \\
\text { - } \text { BI architecture [1] } \\
\text { - Architecture of } \\
\text { mobile cloud } \\
\text { computing [5] [32] }\end{array}$ \\
\hline $\begin{array}{l}\text { Requireme } \\
\text { nt }\end{array}$ & $\begin{array}{l}\text { - ETL } \\
\text { requirement [4] }\end{array}$ & & $\begin{array}{l}\text { - BIaaS requirement [59] } \\
\text { - Requirement for security system } \\
\text { in the cloud [83] }\end{array}$ & $\begin{array}{l}\text { - DSS requirement } \\
\text { [36] }\end{array}$ \\
\hline $\begin{array}{l}\text { Methodolo } \\
\text { gy }\end{array}$ & $\begin{array}{l}\text { - Methodology } \\
\text { for data } \\
\text { integrity } \\
\text { verification } \\
\text { [37] [43] }\end{array}$ & & & $\begin{array}{l}\text { - Methodology for BI } \\
\text { migration [37] [38] }\end{array}$ \\
\hline Algorithm & $\begin{array}{l}\text { - Intercloud data } \\
\text { transmission } \\
\text { algorithm [44] }\end{array}$ & $\begin{array}{l}\text { - Algorithm for } \\
\text { building parallels } \\
\text { cubes based on } \\
\text { MapReduceMerge } \\
\text { [54] } \\
\text { - Algorithm to } \\
\text { process multiple } \\
\text { join queries in the } \\
\text { cloud [55] } \\
\text { - Resources planning } \\
\text { algorithm [56] } \\
\text { - Algorithm for } \\
\text { building cube with } \\
\text { MapReduce [57] } \\
\text { - Aggregate query } \\
\text { algorithm [58] }\end{array}$ & & \\
\hline
\end{tabular}




\begin{tabular}{|c|c|c|}
\hline $\begin{array}{l}\text { Implement } \\
\text { ed system }\end{array}$ & $\begin{array}{l}\text { - Tool for analysis in the cloud } \\
\text { [67] } \\
\text { - Tool for online aggregation in } \\
\text { the cloud [61] } \\
\text { - Tool for scalable runtime } \\
\text { optimized of cloud data analytics } \\
\text { [73] } \\
\text { - Tool for incorporating BI in } \\
\text { cloud market [78] } \\
\text { - Tool to maintain the elastic } \\
\text { OLAP cloud platform [62] } \\
\text { - Tool for application } \\
\text { reconfiguration [71] } \\
\text { - Tool to combine the scale of } \\
\text { Google App Engine with offline } \\
\text { data analytics [72] }\end{array}$ & \\
\hline Example & - Example of building OLAP on & - $\quad$ Example of \\
\hline
\end{tabular}




\begin{tabular}{|l|l|l|l|l|}
\hline & & cloud [52] & security measures & {$[40]$} \\
\hline
\end{tabular}

Table 1: Synthesis of the literature review

As Table 1 shows, some artifacts cover all three steps of BI (BI as a whole). We will describe these artifacts before describing artifacts dedicated to each step of BI.

\subsection{BI as a whole}

Increasingly, mobile is used for BI applications in the cloud. It serves as a data transmission channel to the cloud and from the cloud. Fernando et al. proposed a classification of issues found in this area (taxonomy) [31]. This taxonomy is based on issues related to operational level; end user level; service and application level; privacy, security and trust; context-awareness; and data management. Operational issues are related to the underlying technological matters such as the method of offloading calculations, cost-benefit models that help in making the decision to offload or not, how devices mobility is managed, and how connection protocols are used. End user issues refer to incentives to collaborate, and presentation and usability issues. Service and application issues include fault-tolerance for meeting availability requirements, supporting performance at service level, and cloud APIs. Privacy, security and trust issues pertain to general cloud security, mobile cloud security, and privacy. Context-awareness relates to service provisioning, risk assessment, identifying potential resources, common activities, and energy awareness. Data management issues pertain to personal data storage on mobile cloud, data access issues, data portability and interoperability, and embedded mobile databases.

Khan et al. introduced a graphical representation (architecture) of mobile cloud computing [5]. This architecture includes two main parts: mobile client and cloud service provider. This cloud must be secured to ensure security and privacy protection services. In addition, the cloud provides user management, key management, encryption on demand, intrusion detection, authentication, and authorization services to mobile users. Moreover, secure routing protocols must be used to protect the communication channel between the mobile device and the cloud.

Dinh et al. provided also an architecture of mobile cloud computing [32]. This architecture shows how mobile devices are connected to the mobile networks via base stations (e.g., base transceiver station, access point, or satellite) establishing and controlling the connection (air links) and functional interfaces between the devices. Mobile users' requests and information (e.g., ID and location) are transmitted to the central processors that are connected to servers providing mobile network services. Mobile network operators can provide mobile users with services such as authentication, authorization, and accounting based on the home agent and subscribers' data stored in databases. The subscribers' requests are then delivered to a cloud through the Internet. In the cloud, controllers process the requests to provide mobile users with the corresponding services. These services are based on the concepts of utility computing, virtualization, and service-oriented architecture (e.g., web, application, and database servers).

Several frameworks, architectures, and methodologies are implemented to help organizations that wish to migrate their BI to the cloud. Thus, Verdejo et al. introduced a framework for BI migration to the cloud [33]. This framework supports a migration based on the requirements of the organizations that wish to move their BI system to the cloud premises. There are four main modules in this framework: system modeling module, cloud environments modeling module, migration strategies generators, and migration strategies. The first two components are modeled according to the migration degree that the organization adopts for its BI system. Thus, the organization can choose a partial or a total migration to the cloud. In addition, they can move their BI tools to IaaS or PaaS platforms to leverage the additional scalable resources, 
Or to a SaaS solution and use available BI tools. The migration strategies generator contains a security module and a synchronization module. In order to avoid data from being compromised at the moment of migration, the security module ensures end-to-end encryption. Some data may not leave the premises of the BI-user organization, because of the law or political sensitivity, but may be required by BI tools running in the cloud environment. Through the security module, the elements of these sensitive data are substituted for a generated token that represents the sensitive or secret data. The synchronization module serves as a bridge and controls the communication between all framework components. The migration options are stored as migration strategies.

Baars and Kemper proposed a framework representing the logical structure of cloud BI [34]. This framework contains two mains components: service composition and service distribution. Both components are covered by an umbrella which is a general provider. The composition and the distribution must be done according to the service granularity expected in the contract. There are six scenarii that illustrate the different service organizations existing in the cloud: i) Add-on services scenario: some components (e.g. a web search engine) are selected from the cloud to BI infrastructure; ii) Tool replacement scenario: The cloud makes available a complete tool, for instance a data mart or OLAP tool. This is SaaS (Software as a Service); iii) Solution provision scenario: The cloud supports a software and hardware remote solution; iv) Business network scenario: A solution provider acts within a corporate network. The cloud aspect resides in the abstraction of the physical infrastructure that has become virtual; v) Best-of-breed scenario: The replacement of the tool is pushed to a higher level to the point where all components of the BI infrastructure are provided by an external supplier; vi) BI mashup scenario: The BI solution is freely constituted using a global market space over the Internet.

Kasem et al proposed a blueprint that represents the fundamental organization of BI on the cloud (architecture) [35]. This architecture shows the minimum elements that the cloud can offer to BI. These elements are: i) Hardware processing, storage, and networks. ii) Software: the operating systems and drivers required to handle the hardware. iii) Data integration: the tools performing the ETL and data cleansing processes. iv) Database: the relational or multidimensional database systems that contain the data. v) Data warehousing tools: the set of applications that allow the creation and maintenance of the data warehouse. vi) BI tools: the set of front-end applications that read and analyze data.

Chaudhuri et al. proposed a BI architecture, describing the BI tasks [1]. It is not dedicated to the cloud and it is classically composed of three parts for respectively data collection and blending, data modeling and storage, and analytics.

Jun and Jun introduce a set of requirements to implement a decision support system (DSS) for BI in the cloud [36]. This needs to describe how a decision-making process is articulated. This DSS contains three basic components: a database, a model base, and a user interface. Thus, it requires: i) A database management system (DBMS) or a data warehouse that consists of structured, real-life information, such as customer account records, which provides quantitative analytic information for crucial issues. ii) A model base management system (MBMS), which contains one or more models supporting the analysis that the system will perform. The model base reflects the internal nature of large amounts of data, and enables qualitative analysis to assist decision makers by means of knowledge reasoning. iii) A user interface that provides the decision maker with feedback.

Gash et al. proposed the procedure to follow step by step for BI migration to the cloud (methodology) [37]. This procedure includes four steps. The first is the evaluation of the environment. Organizations that wish to migrate their BI to the cloud must conduct the analysis of the current environment. The second step is the design of a cloud-based solution. In this step, organizations must design a solution to expose their data to the cloud provider by web service or secure tunnel. This solution must take into account the amount of data being transferred, speed of transferring and security concerns on the one hand. On the other hand, they must work with the cloud provider to design the appropriate structure for reporting. The third step is the solution building phase. Organizations must work with the cloud provider to implement the integrations required to expose data to the cloud provider and to create reports to meet the needs uncovered in the evaluation phase. The fourth step is the support phase. In this step, organizations must not only support everyday operations of cloud-based environments, but also monitor its usage.

Muriithi and Kotzé introduced another procedure (methodology) for BI migration [38]. This procedure includes three following steps: i) Situational analysis: this step consists of the current evaluation of an organization to identify potential opportunities for cloud BI. Potential candidates for migration may be hardware or software tools across the three layers of BI: data layer (ETL, data warehouse or data mart), logic layer (e.g. reporting tool, OLAP tool or data mining tool) and access layer (e.g. a portal or a mobile 
application). ii) Suitability assessment: in this step, each candidate to migration must be evaluated for cloud suitability subjects. The evaluation criteria include business value, technical viability, risk exposure, and

organizational impact. iii) Implementation: BI tools that pass the suitability assessment are migrated to the cloud and their performance will be evaluated to determine how well they meet the needs of the organization.

BI Research also involves taking into account specific aspects such as social media. As an illustration, Dinter and Lorenz introduced a framework for a Social BI Research Agenda, enabling to guide the derivation of a social BI research agenda [39]. This is a matrix which crosses two dimensions: social media characteristics and BI design areas. Social media characteristics are highly dynamic data, high data volumes, semi-structured or unstructured data, extensive meta-data, unknown data quality, wisdom of the crowds, user network information, unclear legal situation, and coverage by literature. BI areas considered are

the following: users and customers, products and services, process, data (architecture and development, security management, quality management, and meta-data management), information and communication technology (ICT), techniques, governance, and strategy.

Cloud computing raises several issues, such as security. Research on cloud computing also focuses on security concerns in order to strengthen the security of all resources in the cloud. Ryan suggests an example to put forward the safety concern when exchanging cloud data [40]. As a real life example, EasyChair and EDAS systems allow a conference chair to create the conference account in the cloud. They handle all the necessary administration such as assignment of papers to program committee members, collection and distribution of reviews and discussion. In addition, these systems produce emails to authors and reviewers and reports such as acceptance statistics. Cloud providers take responsibility for the data across all conferences. They are custodians of a huge quantity of data regarding the submission and reviewing behavior of thousands of researchers, aggregated across multiple conferences. Thus cloud-computing-based conference management generate privacy concerns. Ultimately, technological solutions which would provide the data owner with verifiable guarantees that their data remains confidential are required.

\subsection{Data collection and blending}

Negash proposed a data framework for BI [41]. This framework represents the logical structure to perform the integration of structured and semi-structured data. It includes three main components: i) acquisition, integration, and cleanup of structured data; ii) acquisition, integration, and cleanup of semistructured data; iii) search, analysis and delivery. This BI framework shows that semi-structured data and structured data are equally important to help decision makers. Moreover, the processes of acquisition, cleaning, and integration are required for both structured and semi-structured data.

A cloud data warehouse includes not only organizations' local data, but also data from other clouds. Therefore, ETL is also used to exchange data between different clouds. Clay et al. proposed a generic framework called RHIC (Resource Harvester for Interactive Clouds) [42]. This framework autonomously optimizes a hybrid cluster running with residual resources. RHIC provides intelligent cluster sizing for a wide range of throughput-oriented parallel batch workloads. It combines online profiling with periodic job progress and system resource monitoring.

$\mathrm{BI}$ in the cloud currently offers one-way data integration (from user to cloud) via Extract-TransformLoad (ETL) tools. In [4] Herwig proposed additional functionalities (requirement) in order to enable the ETL not only to perform integration but also to compute some results and send them to user interfaces. The interface definition must include the data to be exchanged, the data format, and the communication (protocol level). Regarding security concerns, only those end-users of the cloud-based application are allowed to see the report of this application. Before the cloud-based application can request data from the cloud-based BI solution, it has to authenticate itself.

In [43] Chang et al. proposed a methodology for verifying the integrity and confidentiality of data throughout the ETL step till storage in the data warehouse. The verification of integrity regarding remote data contains the followings steps: setup and data upload; authorization for Third-Party Auditor (TPA); integrity proof; updated data; updated metadata; and verification of updated data. In setup and data upload, the client will need to prepare verification metadata in order to verify the data without retrieving them. In authorization for TPA, there are three parties (client, auditor and storage service provider). This step is required when users need a semi-trusted TPA to verify the data on their behalf. Challenge and verification of data storage is where the integrity verification has to be fulfilled. Data update is required in dynamic data contexts. In some cases, the client needs to perform updates to some of the cloud data storage. If the data is stored in blocks of varying size for efficiency reasons, there will be more types of updates to address. In metadata update, the client will need to update the verification metadata with the existing keys so that the data storage stay 
verifiable without retrieving all the data stored and/or re-running the entire setup phase. In verification of updated data, the client verifies the data update process in order to see if the updating of both user data and verification metadata has been performed successfully in order to ensure that the data can still be verified correctly in the future.

Hyuck et al. proposed an inter-cloud data transmission algorithm that applies a filter each time after reading data [44]. All data files are uploaded to HDFS (Hadoop Distributed File System). Before transmission between clouds, data will be filtered. Input data are recognized as sets of tuples, and sent to the client side applying filters. The client side then receives filtered data.

\subsection{Data modeling and storage}

Data collected from different sources must be modeled and stored in a data warehouse. The data warehouse is the central element of BI. BI in the cloud provides three possibilities to implement and manage a data warehouse. The first occurs when an organization already has a data warehouse and plans to transfer it to the cloud. The second is to create a data warehouse directly in the cloud and store it in the cloud. Finally, the third situation is that of an organization that manages its data warehouse on its own servers but solicits cloud data warehouse tools.

Chi et al. introduced a data structure (system design), called SLA-tree, to support profit-oriented decisions in cloud computing [45]. This model consists of multiple servers, where queries arrive to the system dispatcher. The dispatcher sends each incoming query to one of the servers or may reject the query if admission control is in place. When a query is dispatched to a database server, if the server is busy, the query becomes part of a buffer of queries waiting to be executed. When the execution of the current query in the server is completed, the query at the front of the buffer is executed next, or optionally, a scheduler picks a query from the buffer to be executed next according to certain scheduling policies.

Tapiador et al. proposed a framework that allows the hypercube generation to be easily done in a MapReduce infrastructure [46]. This framework has been implemented in Java considering the following features: i) A thin layer on top of Hadoop that allows users or external tools to focus only on the definition of the hypercubes to compute. ii) Hide the complexity of this novel computing paradigm and the distributed system on which it runs. Therefore, it provides a way to deal with a cutting-edge distributed system (Hadoop) without any knowledge of big data internals. iii) Possibility to process many hypercubes in one single scan of data, taking advantage of Hadoop jobs, thus reducing the time for generating the precomputed statistics required for each data release. iv) Leverage the capabilities offered by this new computing model so that the solution is scalable. v) Java generics have been used throughout the framework in order to ease its integration in any domain.

Tancer and Varde introduce the medical markup language (MML) for storing and analyzing medical data using cloud services [47]. MML allows quick and easy distribution of medical data over the Web without requiring conversion from relational formats. The head of an MML document contains two sections: the clinical document header and the body. The clinical document header holds information about the document type, origination creation date, healthcare provider information, basic patient information and the local header. An MML document is a container for medical data to be transferred from one health institution to another, even if both institutions implement different systems. The cloud is an appropriate solution for using MML for medical data storage and analytics worldwide.

Organizations that choose to migrate to the cloud can also take advantage of large amounts of data (big data) contained in the cloud. According to the sensitivity of their data, they will opt for migrating a part of or their entire data warehouse. The cloud offers virtual spaces that seem unlimited for customers.

Qian et al. provided a cloud computing architecture [48]. This architecture has three layers: resource, platform, and application. Resource is the infrastructure layer that includes physical and virtualized computing, storage and networking resources. Platform is the most complex layer that could be split into many sub-layers, especially a storage sub-layer providing unlimited storage and caching capability. An application layer supports large and distributed transactions and management of huge volumes of data. These three layers provide external service through web services or more secured networks.

In order to take advantage of the cloud, a data warehouse must have a structure that matches the cloud functionalities. To achieve this, in [49], Kaur et al. suggested a cloud data warehouse architecture. This architecture combines the traditional data warehouse architecture with the description of the various services provided by the cloud. It identifies different layers that may be hosted in the cloud. The data staging or inbound layer houses the extraction programmers. They can be hosted in the cloud by initiating the 
appropriate data governance model at the data source layer itself. They use the extraction logic to cleanse and summarize the data. Complete data warehouses can be designed in the cloud with the use of Data as a Service (DaaS) and simply paying on a "pay-per-use" basis. On-demand servers for storage also provide scalability.

In [50] AbdelBaky et al.introduced an objective-driven architecture called MapReduce-CometCloud. This architecture allows distributing data chunks to nodes and then pushes computational tasks to the nodes that contain these chunks in order to achieve the best performance in computing. It includes four MapReduce components: i) Job submission and monitor provide facilities to read input files through Input Reader. ii) Mapper that takes a set of data and transforms it into a list of key/value pairs. The Mapper outputs are sorted and then partitioned per Reducer. Users can optionally specify a combiner to perform local aggregation of the intermediate outputs, which helps to cut down the amount of data transferred from the Mapper to the Reducer. iii) Reducer takes the list that resulted from the map function and reduces the list of key/value pairs based on their key. The number of output is the same as the number of reduce tasks. iv) Output Collector collects data output by the Mapper or the Reducer (either the intermediate outputs or the output of the job).

In [51] Cao et al. proposed an architecture of a system able to support both data-intensive analytical operations (OLAP) and online transactions (OLTP). This is an elastic data storage system, which is designed to support both functionalities within the same storage. There are three major modules in this architecture: data import control, data access control, and physical storage. The data import control module supports efficient data bulk-loading from external data sources. The data import control module consists of two subcomponents, namely import manager and write cache. The import manager has separate protocols to communicate with different data sources. The write cache resides in memory and temporarily buffers the imported data that are eventually flushed to the physical storage when the write cache is full. The physical storage module contains three main components: distributed file system (DFS), meta-data catalog and distributed indexing. The DFS is required when the imported data are actually stored. The meta-data catalog maintains the meta-information about the tables in the storage. Distributed indexing maintains various distributed secondary indexes over the data stored in DFS. The data access control module is responsible for performing data access requests from both OLAP jobs executed by the elastic execution engine and OLTP requests submitted by end users.

In [52] Arres et al. implemented an architecture for building OLAP cubes in the cloud and an example illustrating the usefulness of this architecture. The latter is built using the Hive DBMS in a Hadoop environment. It illustrates the partitioning of the data warehouse on different clusters (nodes), the construction and the querying of OLAP cubes by users. The implemented example includes two phases: i) Construction phase of the data warehouse. The latter is implemented using the star schema model of sales stores. ii) Loading phase and OLAP cube construction. This phase builds a data cube answering a question requiring multi-level aggregation.

Palanisamy et al. provided a system architecture called CURA for data analytics in the cloud [53]. This architecture creates the best cluster configuration for the jobs using MapReduce. It is composed of the following components: i) Secure instant VM allocation employs a secure instant VM allocation scheme that reduces response times for jobs, in contrast to existing MapReduce services that create VMs on demand. ii) Job Scheduler is faced with the challenge of scheduling jobs among available VM pools while minimizing global cloud resource usage. Therefore, carefully executing jobs in the best VM type and cluster size among the available VM pools becomes a crucial factor for performance. The scheduler has knowledge of the relative performance of the jobs across different cluster configurations from the predictions obtained from the profile and analysis service. iii) VM Pool Manager deals with the challenge of dynamically managing the VM pools to help the job scheduler effectively obtain efficient resource allocations. In addition, this component may perform optimization such as power management by suitably shutting down VMs.

In order to improve more analysis in the cloud, many algorithms are proposed. In [54] Wang et al. proposed four algorithms for building parallel data cubes based on the MapReduceMerge data processing system. These algorithms reduce data movement compared to traditional MapReduce, and make the data storage more efficiently to support the OLAP tasks. In MapReduceMerge, the map function is identical to the original MapReduce, but there is a difference between the reduce functions. The reduce function of MapReduceMerge produces a key/value list instead of just values. Each of these algorithms performs a defined task. The first algorithm (merge control flow) divides the entire key into different partitions which leads to a Cartesian product between reducers. The second algorithm (k-select) is responsible for matching reducer and merger and obtaining the best performance tradeoff. The third is the data iterator. This algorithm is a data process controller. The last algorithm (merge) determines the merge action and the format of results. 
In cloud data warehouses, data are distributed to different nodes depending on the availability of resources. The location of data can change dynamically. A large volume of data requires increasing the number of nodes and, therefore, increasing the flow of communication messages between nodes. All these factors increase the use of the bandwidth and possibly degrade performance. To remedy this situation, in [55], Kurunji et al. proposed an algorithm to process multi-join queries in the cloud and reduce communication costs. A reference graph is built to help run this algorithm. This reference graph consists of tables and relationships between the tables of the star schema. The inputs of this algorithm are query and reference graphs, and the result of the query is the output. In this algorithm, all the tables in the "from" clause of the query are retrieved and sorted by the corresponding value in the reference graph. Then, the process of each table predicate starts from the first table of the above sorted order.

In [56] Lee et al. introduced a Resource Planning algorithm for video analytic. This algorithm provides the best video processing performance under user defined constraints in a resource shared cloud environment. The inputs of this algorithm consist of the kind of analytic processing that should be performed, the video dataset, and constraints such as time threshold and maximum cost. Output files in nodes are downloaded to the video bank or to other nodes for performing computation tasks automatically.

In [57] Abelló et al. provided three algorithms for building cubes with MapReduce. Corporate historical data are stored in a cloud using BigTable. MapReduce is used as an agile mechanism to deploy cubes in ad-hoc Data Marts. The first algorithm is called Filtered Source Scan. The inputs of this algorithm are the names of the BigTable source and measure table, and the output value in this case is just the key in the source table. The second algorithm is called Indexed Random Access. Its inputs are name of source and index BigTable, and file containing the slicers. Its output is a data cube. The third algorithm is called Indexed Partial Scan. Its inputs are name of source and index BigTable, and file containing the slicers. The output is a data cube. This third algorithm is a mix of the two others. The aim is to use the index but avoid random access to the source.

In [58] Hua et al. introduced an aggregate query algorithm in R-tree structure. The inputs of this algorithm are dimensional attributes and the outputs are aggregate cells. This algorithm is an on-line rankingbased (top-k) aggregate query which can return top-k query results. It creates sorted lists for ranking cuboids and then initializes these sorted lists by scanning materialized cuboids into memory.

\subsection{Analytics}

The analytics step uses the data stored in the data warehouse to perform analysis. There are two possibilities for BI in the cloud: cloud can provide analytical tools as SaaS or organizations can perform themselves their analysis in the cloud using PaaS.

In [59] Chang proposed a new concept called BIaaS (Business Intelligence as a Service). It is a type of SaaS which focuses on how the application offers quality services in cloud environments. BIaaS is a Cloud based service designed to improve the accuracy and quality of both pricing and risk analysis in financial markets, compared with traditional desktop technologies. Chang suggested functionalities for BIaaS (requirement). With the advent of cloud computing, it becomes important to implement models and methods to improve BI tasks in order to perform accurate and fast simulations. Requirements for BIaaS are based on reputable models, compute and track volatility, accuracy, and diversity. Regarding the reputable models, BIaaS adopts reputable models including the Heston Model and visualization APIs to compute the best pricing and risks for different scenarios. Moreover, BIaaS can track the movement of volatility that can help investors to make better judgment for investment when prices are high and volatility is low. Regarding accuracy, BIaaS can compute pricing and risk values to several decimal places and also calculate their mean, lower and upper range to get results that are as accurate as possible. Regarding diversity, BIaaS should not just limit its operations on desktop or a particular platform, but extend to different types of clouds.

In [60] Chen et al. proposed a new concept called CaaaS (Continuous analytics as a Service), dedicated to real-time analytics. More and more applications are dependent on analytics results in real time events. CaaaS is a cloud computing model enabling convenient, on-demand network access to a shared pool of event analytics results. The support of CaaaS is characterized by the following: i) a unified query model over both stored relations and dynamic streaming data, and techniques to extend query engines to support the unified model; ii) the "table-ring" mechanism which allows the infinite analytics results to be kept in a list of small-sized tables based on time sequence, and staged through "switching labels" without actual data copying and moving; iii) CaaaS is scaled out with multiple engines cooperating based on the common data chunking criterion, on both parallel database and network distributed Map-Reduce infrastructures. 
This third stage of BI provides all the data analysis capabilities and supports the distribution of knowledge relevant to different users. The essential elements of this layer are reporting tools, data mining and OLAP (On-Line Analysis Processing). OLAP is based primarily on exploration operations. Given this, several models, methods and tools are implemented for data analysis in the cloud.

Yantao et al. introduced a MapReduce-based online aggregation system (an architecture and implemented system) called COLA [61]. The architecture contains four modules: User Interface, Query Engine, Online Aggregation Executor, and Data Manager. i) User Interface: this module provides interactive and flexible interfaces, users can issue SQL queries or submit MapReduce program via a shell interface. ii) Query Engine: this module is responsible for compiling the SQL query into a directed acyclic graph of MapReduce jobs, and translating the non-OLAP jobs into an online version. iii) Online Aggregation Executor: this module is the key module of COLA performing the online query processing algorithm over MapReduce. It processes the sample data, produces approximate answers with their associated confidence intervals and progressively refines the answers. iv) Data Manager: this module makes use of HDFS to store and manage data. It stores metadata such as mappings between tables and HDFS directories in the Metadata manager that can be used to perform query optimization and compilation in the SQL2MR Translator. The implemented tool provides progressive approximate aggregates for single tables and for multiple joined tables with novel techniques supporting incremental and continuous computing of aggregation, and minimizing the waiting time.

Brezany et al. proposed an architecture and a tool (implemented system) to maintain the elastic OLAP cloud platform [62]. This system provides dynamic provision and extension by utilizing the virtualization of cloud computing platforms such as Amazon EC2. The architecture of the platform consists of several services and brokers utilized to manage and assign free virtual cube servers and cube host resources. The platform involves the resources, described as follows: i) Virtual cube server is a container which can manage one or more virtual cubes. ii) Host cube is the physical storage and computational resource in which multidimensional data arrays are stored and OLAP queries processed. iii) Virtual cube broker is responsible for managing free virtual cube servers. When a client requests a virtual cube server, it should first ask the broker and the broker then assigns a suitable free virtual cube server to the client. Cube host broker aims to manage free cube host resources.

In [63] [64] Al-Aqrabi et al. proposed the BI and OLAP framework which allows multiple OLAP application servers applying parallel query loads on an array of servers hosting relational databases. This framework has seven components: i) a user interface layer that contains a large library of dashboards for graphical reporting; ii) a layer for data analytics that contains different scenarios, reports, stored queries and data models; iii) a layer for storing the OLAP cubes formed by multi-dimensional data extraction from the data layer (the data warehouses); iv) a data integration layer for identification, cleaning, organizing and grouping of data extracted from the data warehouses before the cubes are formed; v) a data layer comprising the data warehouses; vi) a layer for acquiring data from the business processing, decision support and transactional databases used by various functions of the organization; vii) a layer comprising the IT infrastructure components and related resources (data processing, storage, andnetworking).

Wlodarczyk et al. proposed a framework for designing and deploying cloud-based data analytic systems [65]. This framework is based on Hadoop and uses the Web Ontology Language (OWL). It is composed of four layers: knowledge, deployment, processing, and data. The knowledge layer provides several ontologies to completely specify the functionality of a Hadoop-based system described using the taskmethod decomposition technique. In the deployment layer, several specialized processes are used to control both the deployment of the system on the cloud and its monitoring. In the processing layer, the DataStorm framework is explained using a simple algorithm. Its goal is to show how to use basic ontological elements introduced in the Knowledge Layer. The Data layer contains data of different formats.

Chaisiri et al. proposed a framework called CODA (Cloud-Oriented Data Analytics) [66]. This framework includes functions for composing, managing, and processing workflows for big data analytics in cloud computing. This framework contains four layers: workflow client, workflow management, tools and services, and computing resource. The workflow client layer provides the environment where the users compose workflows through workflow composers. A workflow composer prepares tasks (i.e., reusable software components) and each task performs a specific function i.e., reading the data stored in an Excel file, clustering the data with the k-means method, and writing the result to a file. This framework can support different workflow composers e.g., RapidMiner, Taverna, and JOpera. The workflow management layer provides main functions to support the execution of data analytics workflows: Scheduler, monitoring, security, data locality, provisioning, workflow recommender, and visualizer. The tools services layer 
provides the programming tools (or frameworks) and reusable services for creating an executable code of tasks (i.e., software component) that can be used in the workflow composition. The computing resource layer provides computational platforms that host executable codes of tasks.

Soumya et al. proposed an architecture to be deployed in analytical processing based on OLAP tools in a cloud computing environment [67]. This architecture deals with issues such as data warehouse creation, dispatching of data in distributed data centers and materialized view creation. It contains two main modules: ETL and analyzer. ETL is an important module to create the OLAP data servers and to take care of a large number of activities such as data collection, data cleaning, data loading, and data blending. The Analyzer Module ensures faster processing of data. The diverse queries of clients are analyzed through the Query Analyzer. There are already a number of techniques to analyze the users' queries or requirements. Based on the result of the analysis, the materialized views are formed, thanks to the Materialized View Generator module.

Patel et al. proposed an architecture and a tool called P2RAC (implemented system) for data analytics in the cloud [68]. This architecture allows the analyst to perform a simulation or an optimization job. This system comprises three components: core tools, diagnostic tools and configuration files. The core tools provide the functionalities for cluster management, data management and execution management of a task on the Amazon cloud. Regarding diagnostic tools, the P2RAC currently offers two tools, which are used for listing the clusters created by the analyst on the Amazon cloud and for accessing the master instance of a cluster respectively. Configuration files include three files that support the core and diagnostic tools which reside on the analyst site.

Lian and Li Da proposed a taxonomy of algorithms for data mining [69]. The purpose of this taxonomy is to introduce the fundamental methods to decision makers, and let them choose the proper method for their data mining applications. This taxonomy contains two major groups: supervised learning methods and unsupervised learning methods. The supervised learning methods for building a model to predict a specified unknown attribute according to the observed attributes. They can be used for nominal prediction, while only neural networks, regression models, and the nearest neighbor method can be used for numeric prediction. The supervised learning methods include the following algorithms: i) Decision Tree; ii) Bayesian Statistics; iii) Neural Network; iv) Support Vector Machine; v) Regression; vi) Nearest Neighbor. The Unsupervised learning methods for extracting patterns, such as clusters, process graphs, and correlated items from data. With respect to supervised learning, more accurate prediction is the golden goal. The unsupervised learning methods include itemset mining and clustering. This taxonomy is not specific to BI on the cloud, but all these data mining algorithms may be used on the cloud.

Talia proposed Data Mining Cloud Framework's graphical programming interface [70]. This framework includes the following components: i) a set of binary and text data containers (Azure blobs) used to store data to be mined (input datasets) and the results of data mining tasks (data mining models); ii) a task queue that contains the data mining tasks to be executed; iii) a task status table that keeps information about the status of all tasks; iv) a pool of $\mathrm{k}$ workers, where $\mathrm{k}$ is the number of virtual servers available, in charge of executing the data mining tasks submitted by the users; v) a website that allows users to submit, monitor the execution, and access the results of data mining tasks. This workflow-based data mining framework runs on cloud platforms and uses a service-oriented approach. It offers a flexible programming model, distributed task interoperability, and execution scalability that reduces data analytics completion time.

In [71][84] Goncalves et al. introduced a tool called AWARD (Autonomic Workflow Activities Reconfigurable and Dynamic) which allows more flexibility according to the application characteristics (implemented system). This tool proposes four activities: split, mapper, merger, and reducer. The Split activity gets the size of the input file and calculates the file offsets of each split to be processed by mappers. The Mapper activity invokes the map function for each data record extracted from the input split and the map function stores key/value pairs by invoking the method collect of the MRcollector argument. The Merger activity produces a tuple space identified in parameters with an ordered partition of keys. The Reducer activity produces a tuple space identified in parameters with the final key/value pairs, whose values are reduced using multithreading.

Chohan et al provided a tool (implemented system) [72]. This tool allows combining the scale of Google App Engine (GAE) with the flexibility of customizable offline data analytics. The Task Queue API facilitates the use of multiple, independent user defined queues, each with a rate limit of 100 tasks per second in GAE. A task consists of an URL application, which is called by the system upon task queue. This tool considers two approaches to data synchronization: bulk and incremental data transfer. For bulk transfer, GAE currently provides tools as part of its software development kit (SDK) to upload and download data into 
and out of the GAE data store. AppScale is extended with similar functionality. Incremental transfer implements an asynchronous URL Fetch call to the AppScale analytics cloud for the application upon each destructive update. If this call fails, the GAE and AppScale replicas will be inconsistent until the next time the same entity is updated.

Barga et al proposed an architecture and a tool (implemented system) for a scalable runtime optimized of data analytics in the cloud [73]. The architecture is composed by two Windows Azure worker roles: master and slave. For a given deployment, there will be a single instance of the master and any number of slave instances depending on the deployment configuration. Similar to other MapReduce runtimes, the master performs the scheduling of applications and tasks and handling failures, whereas the slaves simply execute Map and Reduce tasks assigned to them by the master. The communications between master and slaves and between slaves happen directly via TCP connections. This tool called Daytona is a highly optimized iterative MapReduce runtime designed for the cloud.

Mian et al. implemented a framework to solve the problem of provisioning resources in a public cloud [74]. This framework is composed of two main modules: a provisioner and a scheduler. The provisioner takes a description of the workload from the client as input. The description includes the set of query types, the frequencies of each type, the data partition, and the required service level objective (SLO) for each query class. The Scheduler accepts requests from application clients and directs requests to the appropriate VMs based on the query class of the request. The Scheduler can be replicated as needed to ensure that scheduling does not become a bottleneck.

Yuan and Herbert proposed a framework for pervasive healthcare that tries to change the healthcare delivery model [75]: from doctor-centric to patient-centric, from acute reactive to continuous preventive, from sampling to monitoring. On the cloud server, blob storage, multiple queues and worker roles are deployed for the purpose of analyzing users' activity data and producing the best classification model for each user. According to this model, this framework consists of five types of queues: data queue, result queue, register queue, task queue, and model queue. i) Data queue is a general queue and is used to communicate between the client and the controller node. ii) Result queue: When the most suitable model is selected by the allocated worker role (Evaluation Node), the URL of the best model is added to that user's Result Queue. Unlike the Data Queue which is unique and all the users have access to it, one Result Queue is independently created for each individual and doesn't provide public access. iii) Register queue: It is used to transfer the information of a new user. Once a new user is registered, the system assigns him/her a dedicated Result Queue and a blob container. iv) Task queue: In order to make all worker roles start building models in parallel, there should be separate queues. The queues are dedicated to each of the worker roles. v) Model queue: Once the model of each classifier is retrained and evaluated, the evaluation result will be sent to the evaluation node in the model queue.

Shanks et al. suggested a framework for understanding business analytics technology and capabilities that can bring better performance to organizations [76]. This framework contains three components: business analytics resources, value creating action, and firm performance. The business analytics resources component includes business analytics capabilities allowing to identify opportunities. The value creating action component evaluates these opportunities. Finally, this value-creating action may generate firm performance.

Leimeister et al proposed a business process model (system design) for a generic value network of cloud computing [77]. This model is created by providing services that are valuable for other participants of the network. It describes the interactions between different business partners to jointly develop and manufacture a product or service. The manufacturing process is decomposed into its strategically relevant activities, thus determining how competitive advantages can be achieved. Competitive advantages are achieved by fulfilling the strategically important activities cheaper or better than the competition. The different partners considered in this business process are the following: customer, service provider, infrastructure provider, aggregate services provider, and platform provider.

Gatzioura et al focuses on implementation of a business model process (system design) and a tool (implemented system) for incorporating business intelligence in cloud marketplaces [78]. Based on the individual requirements and using profile of each particular cloud customer, this system allows answering the question: "Which offering should I buy"? The user enters his business parameters of interest (which may be availability, security level, price, reliability, product rate, with or without quality assurance, eco-friendliness) and imposes threshold values on them (minimum, maximum or equal), which delimit the possible final solutions' set. 
Aceto et al. proposed a taxonomy of the main aspects of cloud monitoring [79]. This taxonomy consists of four aspects: basic concepts, properties, need for monitoring, and open issues. The basic concepts include a number of concepts at the basis of cloud monitoring that are used to set the context such as: layers, abstraction levels and metrics. Regarding the properties, in order to operate properly, a distributed monitoring system is required to have several properties including scalability, elasticity, adaptability, accuracy, and availability. The need for monitoring is a task of paramount importance for both Providers and Consumers. On the one side, it is a key tool for controlling and managing hardware and software infrastructures; on the other side, it provides information and Key Performance Indicators (KPIs) for both platforms and applications. It includes several activities such as: capacity and resource planning, capacity and resource management, data center management, service level agreement (SLA) management, billing, performance management, and security management. Regarding open issues and future directions, the infrastructure of a cloud is very complex, requiring more effort for management and monitoring. The greater scalability and larger size of clouds compared to traditional service hosting infrastructures involve more complex monitoring systems, which therefore have to be more scalable, robust and fast. Such systems must be able to manage and verify a large number of resources and must do it effectively and efficiently. It must also be able to quickly spot and report performance impairments or other issues, to ensure timely interventions such as the allocation of new resources.

Demirkan and Delen introduced an architecture of service oriented decision support system (DSS) on the cloud [80]. This architecture is composed of three modules: data service, information service, and analytics service. Data-as-a-service began with the notion that data quality could happen in a centralized place, cleansing and enriching data and offering it to different systems, applications or users, irrespective of where they were in the organization, computers or on the network. Information-as-a-Service is a comprehensive strategy for the delivery of information obtained from information services, following a consistent approach using SOA infrastructure and/or internet standards. The concept of analytics-as-a-service (AaaS) referred to agile analytics. It is a comprehensive strategy for the delivery of information obtained from information services, following a consistent approach using SOA infrastructure and/or Internet standards.

Kowalczyk et al proposed a framework that combines a selection of process outcomes, as well as process characteristics that are considered as relevant in managerial decision process research [81]. The framework considers the following decision process phases: identification, development and selection, and focus on the following attributes for each of the three phases: i) Information quality: One of the major benefits of business intelligence and analytics (BI\&A) systems is the provision of accurate, high-quality information which is easily accessible. ii) Comprehensiveness and procedural rationality: In managerial decision process research, procedural rationality describes the level of reliance upon analysis of information in decision making and comprehensiveness characterizes the extent to which analysis is exhaustive within the decision process. iii) Speed: Time savings are another major benefit that is proposed to be realized by BI\&A systems. iv) Phase outcomes: The final decision is not the only result, within a decision process. Each phase produces results, which can be analyzed with respect to their quality and quantity. v) Decision result: The decision result is the outcome of the overall process. vi) Total decision speed: The total decision speed characterizes duration of the overall decision process, which is expected to be reduced.

As in the other stages of BI, security remains a major challenge to be addressed in analyzing data Subashini and Kavitha introduced a process model (framework) for security in the cloud [82]. This model contains three layers: lower, higher and middle. The lower layer represents the different deployment models of the cloud, namely private, community, public and hybrid cloud deployment models. The higher layer represents the different delivery models that are utilized within a particular deployment model. These delivery models are the SaaS, PaaS and IaaS. These delivery models form the core of the cloud and they exhibit certain characteristics like on-demand self-service, multi-tenancy, ubiquitous network, measured service and rapid elasticity which are shown in the top layer. The middle layer describes security. The fundamental elements of the cloud require security which depends and varies with respect to the deployment model that is used, the way by which it is delivered and the character it exhibits. Some of the fundamental security challenges are data storage security, data transmission security, application security, and security related to third-party resources. This involves the use of strong network traffic encryption techniques such as Secure Socket Layer (SSL) and Transport Layer Security (TLS) for security.

$\mathrm{Ng}$ et al introduced architecture and design principles (requirement) for a system that enables secure and privacy preserving data analytics services in the cloud [83]. This architecture includes five main components: security manager, stream manager, Qos (Quality of Service) controller, privacy controller, and 
query processor. The security manager and privacy controller reply for the majority of all privacy and security requirements. The security manager is responsible for achieving standard safety principles such as encryption, decryption and authentication. The privacy controller is the core of the privacy policy enforcement unit. This system has several functionalities including: i) providing a matching mechanism between privacy policy and user preference; ii) controlling the stream manager to examine the set of queries for each purpose to determine if any information is being collected but not being used. About design principles of this system, $\mathrm{Ng}$ et al proposed: i) Purpose specification that states the purpose for which the data is being collected. ii) Consent guarantees that the purpose for which the data is collected has the consent of the user. iii) Limited collection: data collection should be limited to the minimum amount of data satisfying the user's specified purposes. iv) Limited use ensures that the data is used by queries that do not violate the purposes of the collected data. v) Limited disclosure: the system is not allowed to release any information to a third party that is outside the system without the owner's approval. vi) Limited retention: the immediate deletion of user's data once the associated purpose is fulfilled. vii) Accuracy that corresponds to how accurate and up to date is the information maintained by the system.

\section{Research opportunities}

In this section, we define DSR research opportunities related to BI on the cloud. Based on the analysis of Table 1 resulting from our SLR and the different dimensions of our framework, we suggest some new research avenues.

We organize our discussion according to the steps of BI, as proposed in our framework. Table 2 proposes new artifacts that could bring added value to the field of BI in the cloud. We explain below how we built this table. We collected, in the papers of our SLR, the different contributions imagined by researchers in their future research. They are precious indicators, since they are generally the next steps of research-inprogress. In this sense, it is highly probable that they are feasible and even that they will soon appear in future papers. Table 2 summarized research opportunities identified in the literature and research opportunities identified by us, based on our typology of DSR artifacts. Our proposal appears in bold.

\begin{tabular}{|c|c|c|c|}
\hline & $\begin{array}{l}\text { Data collection and } \\
\text { blending }\end{array}$ & $\begin{array}{l}\text { Data modeling and } \\
\text { storage }\end{array}$ & Analytics \\
\hline Meta model & \multicolumn{3}{|c|}{ Multidimensional meta-model } \\
\hline System design & & & - Business process \\
\hline Ontology & $\begin{array}{l}\text { - Ontology for data } \\
\text { integration }\end{array}$ & & \\
\hline Taxonomy & \multicolumn{3}{|c|}{$\bullet \quad$ Taxonomy of BI migration } \\
\hline Methodology & $\begin{array}{l}\text { - Methodology to develop } \\
\text { tools or tool components }\end{array}$ & $\begin{array}{l}\text { - Methodology model and to } \\
\text { store data } \\
\text { - Methodology to develop } \\
\text { tools in the cloud [39] }\end{array}$ & $\begin{array}{l}\text { - Methodology to } \\
\text { develop customer- } \\
\text { oriented applications }\end{array}$ \\
\hline Guideline & $\begin{array}{l}\text { - Guideline to properly } \\
\text { configure cloud resources }\end{array}$ & $\begin{array}{l}\text { - Guideline to properly } \\
\text { configure cloud resources } \\
\text { - Guideline for building tools } \\
\text { in the cloud [84] }\end{array}$ & $\begin{array}{l}\text { - Guideline to properly } \\
\text { configure cloud } \\
\text { resources }\end{array}$ \\
\hline Implemented system & \multicolumn{3}{|c|}{ - Tool to accomplish the BI tasks in the cloud [60] [78] } \\
\hline Example & \multicolumn{3}{|c|}{ - Example of BI migration } \\
\hline
\end{tabular}

Table 2: Opportunities for DSR on BI in the cloud

\subsection{BI in the cloud as a whole}

Regarding BI in the cloud in general, the literature proposed implementation of tools that can enable users to accomplish the BI tasks in the cloud [60] [78]. We propose to define a taxonomy of BI migration, including guidelines to properly configure cloud resources, and examples of BI migration. These guidelines are of great interest for all companies which want to migrate their BI on the cloud. Structuring these 
guidelines thanks to a taxonomy will help us to provide users with a progressive decision process. We also plan to build a practical example based on a real-life case study.

\subsection{Data collection and blending}

Data collection and blending is a tedious task of BI. In order to allow users to properly conduct data collection and blending in the cloud, it is necessary to focus on implementation of the following artifacts:

- Methodologies, in order to enable organizations to develop their customized applications for data collection and blending, or to add components to their applications.

- Ontologies that could facilitate the integration of tabular data and big data. Big data must be added to transactional data for enriching the analysis. Thus, data providers and data consumers must share the same language. Ontologies should facilitate the automation of data integration.

6.3. Data modeling and storage

The literature proposes methodologies to develop tools or tool components in the cloud [39], and guidelines for building tools in the cloud [84] as future research. We propose implementation of:

- Methodologies to store and/or model data in the cloud.

- Meta-model: a multidimensional meta-model, helping to design the cloud data warehouse. We argue that this meta-model, once developed, could enable the instantiation of classical multidimensional models in the specific context of the cloud or to bring new constructs enriching these models.

6.4. Analytics

We propose implementation of following artifacts:

- The definition of a process model (system design) that could contribute to a better understanding of service requests initiated by users.

- Methodologies to enable organizations to develop their customized applications for analysis or to add components to their applications.

\section{Discussion and conclusion}

New research topics frequently emerge from the opportunity to combine new paradigms or new concepts. We identified BI in the cloud as such an emergent technology [85]. We conducted an initial literature review allowing us to confirm the interest of researchers for this topic [2]. In this paper, we performed a systematic literature review (SLR) to ensure more completeness to our study. Based on SLR principles, we limited this review to top journal and conference publications.

The first contribution of this paper is a synthesis of the artifacts produced on this major topic of BI in the cloud. Thanks to our refined typology of artifacts, we produced a comprehensive set of results. In order to help the reader apprehend this richness, we proposed a two-dimensional framework based, respectively, on the artifact typology and the BI steps.

Another contribution is the proposal of research avenues on this topic. We identified these avenues from i) discussions and conclusions proposed by authors in the papers found through the SLR, ii) logic reasoning based on previous findings in other fields.

Our future research will include development of a decision support system helping practitioners to decide if and how to migrate their BI to the cloud. Another research avenue is linked to a systematic study of artifact precedence links. Eliciting strong and weak precedence paths could help researchers to find efficient research plans in DSR. As an example, when a paper describes a set of guidelines to help practitioners or researchers in a given field, a following step could be the definition of a method based on these guidelines and providing practitioners with well-defined steps. 
Besides, we agree that our SLR suffers from some limitations. The false positives have been discarded by our screening step. But, by only performing search by keywords, we may miss some important publications. Moreover, we could not check if all artifacts were really specific or if some papers just adapted previous artifacts to make them available in the viewpoint of BI on the cloud. Conducting again the same SLR in a few months will also help us to measure the productivity of research in this field and check if BI in the cloud is really a new topic.

Acknowledgements. We thank the reviewers for their helpful comments as well as MoBiD 2014 program committee members that have enabled us to improve our paper.

\section{References}

[1] S. Chaudhuri, U. Dayal, V. Narasayya, An overview of business intelligence technology, Commun. ACM. 54 (2011) 88-98.

[2] O. Sangupamba Mwilu, N. Prat, I. Comyn-Wattiau, Business Intelligence and Big Data in the Cloud: Opportunities for Design-Science Researchers. Advances in Conceptual Modeling - Proceedings of ER 2014 workshops. Atlanta, GA, USA, pp.75-84, (2014).

[3] IDC, IDC Cloud, (n.d.).http://www.idc.com/prodserv/FourPillars/mobility/index.jsp.

[4] V. Herwig, Business intelligence as a service for Cloud-based applications, in: Intell. Data Acquis. Adv. Comput. Syst. IDAACS 2013 IEEE 7th Int. Conf. On, IEEE, 2013: pp. 188-192. [5] A.N. Khan, M.L. Mat Kiah, S.U. Khan, S.A. Madani, Towards secure mobile cloud computing: A survey, Future Gener. Comput. Syst. 29 (2013) 1278-1299.

[6] A.R. Hevner, S. Ram, S.T. March, J. Park, Design Science in Information Systems Research, MIS Q. 28 (2004) 75-105.

[7] S.T. March, G.F. Smith, Design and Natural Science Research on Information Technology, Decis. Support Syst. 15 (1995) 251-266.

[8] H. Chen, R.H. Chiang, V.C. Storey, Business Intelligence and Analytics: From Big Data to Big Impact., MIS Q. 36 (2012) 1165-1188.

[9] H. Yang, M. Tate, Where are we at with cloud computing?: a descriptive literature review, in: 20th Australas. Conf. Inf. Syst. Melb., 2009: pp. 2-4.

[10] H.A. Simon, The sciences of the artificial, 3. ed., [Nachdr.], MIT Press, Cambridge, Mass., 2008.

[11] P.B. Goes, Design Science Research in Top Information Systems Journals, MIS Q. (2014) iii-viii.

[12] S. Gregor, D. Jones, The anatomy of a design theory, J. Assoc. Inf. Syst. 8 (2007) 312-335.

[13] P. Offermann, S. Blom, M. Schönherr, U. Bub, Artifact types in information systems design science-a literature review, in: Glob. Perspect. Des. Sci. Res., Springer, 2010: pp. 77-92. [14] B. Kitchenham, P. Brereton, A systematic review of systematic review process research in software engineering, (2013) 2049-2075.

[15] F. Rowe, What literature review is not: diversity, boundaries and recommendations, Eur. J. Inf. Syst. 23 (2014) 241-255.

[16] https://www.google.be/webhp?sourceid=chrome-instant\&ion=1\&espv=2\&ie=UTF$8 \# q=E R A+2012+\% 3 A+$ journal+ranking+list, (2012).

[17] https://www.google.be/webhp?sourceid=chrome-instant\&ion=1\&espv=2\&ie=UTF8\#q=ERA+2012+:+conference+ranking+list, (2012).

[18] T. Davenport, J. Harris, Competing on analytics, harvard business review 84 (2006) 98-107

[19] M. Minelli, M. Chambers, A. Dhiraj, Big data, big analytics - Emerging business intelligence for today's businesses, (2013).

[20] S. Edwards, L. Lavagno, E.A. Lee, A. Sangiovanni-Vincentelli, Design of embedded systems: Formal models, validation, and synthesis, in hardware/software co-design (2001) 86

[21] T.R. Gruber, Toward principles for the design of ontologies used for knowledge sharing?, Int. J. Hum.Comput. Stud. 43 (1995) 907-928. 
[22] R.C. Nickerson, U. Varshney, J. Muntermann, A method for taxonomy development and its application in information systems, Eur. J. Inf. Syst. 22 (2013) 336-359.

[23] CIO Council, Federal enterprise architecture framework, https://www.whitehouse.gov/sites/default/files/omb/assets/egov_docs/fea_v2.pdf(1999). [24] D.E.R. Denning, Information warfare and security, (1999). http://vxheaven.org/lib/pdf/Information\%20warfare\%20and\%20security.pdf.

[25] M. Jarke, P. Loucopoulos, K. Lyytinen, J. Mylopoulos, W. Robinson, The brave new world of design requirements, Inf. Syst. 36 (2011) 992-1008.

[26] J.F. Nunamaker Jr, R.O. Briggs, R.H.S. Gert-Jan De Vreede Jr, Special Issue: Enhancing Organizations' Intellectual Bandwidth: The Quest for Fast and Effective Value Creation, J. Manag. Inf. Syst. 17 (2000) 38.

[27] O. Hanseth, K. Lyytinen, Design theory for dynamic complexity in information infrastructures: the case of building internet, J. Inf. Technol. 25 (2010) 1-19.

[28] E. Kornyshova, R. Deneckère, C. Salinesi, Method chunks selection by multicriteria techniques: an extension of the assembly-based approach, in: Situational Method Eng. Fundam. Exp., Springer, 2007: pp.

[29] S. Purao, V. Vaishnavi, Product metrics for object-oriented systems, ACM Comput. Surv. CSUR. 35 (2003) 191-221.

[30] N. Prat, I. Comyn-Wattiau, J. Akoka, « A taxonomy of evaluation methods for information systems artifacts », accepted for publication in the Journal of Management Information Systems

[31] N. Fernando, S.W. Loke, W. Rahayu, Mobile cloud computing: A survey, Future Gener. Comput. Syst. 29 (2013) 84-106.

[32] H.T. Dinh, C. Lee, D. Niyato, P. Wang, A survey of mobile cloud computing: architecture, applications, and approaches: A survey of mobile cloud computing, Wirel. Commun. Mob. Comput. 13 (2013) 15871611. doi:10.1002/wcm.1203.

[33] A. Juan-Verdejo, B. Surajbali, H. Baars, H.-G. Kemper, Moving Business Intelligence to cloud environments, in: Comput. Commun. Workshop INFOCOM WKSHPS 2014 IEEE Conf. On, IEEE, 2014:

[34] H. Baars, H.-G. Kemper, Business intelligence in the cloud?, In : Communication Software and Networks (ICCSN), 2011 IEEE 3rd International Conference on. IEEE, 2011. p. 650-655.

[35] M. Kasem, E.E. Hassanein, Cloud Business Intelligence Survey, (2014).

[36] L. Jun, W. Jun, Cloud Computing Based Solution to Decision Making, Procedia Eng. 15 (2011) 18221826. doi:10.1016/j.proeng.2011.08.339.

[37] D. Gash, T. Ariyachandra, M. Frolick, Looking to the Clouds for Business Intelligence, J. Internet Commer. 10 (2011) 261-269.

[38] G.M. Muriithi, J.E. Kotzé, A conceptual framework for delivering cost effective business intelligence solutions as a service, in: ACM Press, 2013: p. 96.

[39] B. Dinter, A. Lorenz, Social Business Intelligence: a Literature Review and Research Agenda, Proceedings of the 33rd ICIS (2012).

[40] M.D. Ryan, Cloud computing security: The scientific challenge, and a survey of solutions, J. Syst. Softw. 86 (2013) 2263-2268.

[41] S. Negash, Business intelligence, Commun. Assoc. Inf. Syst. 13 (2004) 54.

[42] R.B. Clay, Z. Shen, X. Ma, Accelerating Batch Analytics with Residual Resources from Interactive Clouds, in: IEEE, 2013: pp. 414-423. [43] C. Liu, C. Yang, X. Zhang, J. Chen, External integrity verification for outsourced big data in cloud and IoT: A big picture, Future Gener. Comput. Syst. (2014).

[44] H. Han, Y.C. Lee, S. Choi, H.Y. Yeom, A.Y. Zomaya, Cloud-Aware processing of MapReduce-based OLAP applications, in: Proc. Elev. Australas. Symp. Parallel Distrib. Comput.-Vol. 140, Australian Computer Society, Inc., 2013: pp. 31-38. [45] Y. Chi, H.J. Moon, H. Hacigümüş, J. Tatemura, SLA-tree: a framework for efficiently supporting SLA-based decisions in cloud computing, in: Proc. 14th Int. Conf. Extending Database Technol., ACM, 2011: pp. 129-140..

[46] D. Tapiador, W. O’Mullane, A.G.A. Brown, X. Luri, E. Huedo, P. Osuna, A framework for building hypercubes using MapReduce, Comput. Phys. Commun. 185 (2014) 1429-1438.

[47] J. Tancer, A.S. Varde, The Deployment of MML for Data Analytics over the Cloud, in: IEEE, 2011: pp. 188-195. [48] L. Qian, Z. Luo, Y. Du, L. Guo, Cloud computing: An overview, in: Cloud Comput., Springer, 2009: pp. 626-631. h

[49] H. Kaur, P. Agrawal, A. Dhiman, Visualizing Clouds on Different Stages of DWH-An Introduction to Data Warehouse as a Service, in: Comput. Sci. ICCS 2012 Int. Conf. On, IEEE, 2012: pp. 356-359.

[50] M. AbdelBaky, H. Kim, I. Rodero, M. Parashar, Accelerating MapReduce Analytics Using CometCloud, in: IEEE, 2012: pp. 447-454..

[51] Y. Cao, C. Chen, F. Guo, D. Jiang, Y. Lin, B.C. Ooi, et al., Es 2: A cloud data storage system for supporting both oltp and olap, in: Data Eng. ICDE 2011 IEEE 27th Int. Conf. On, IEEE, 2011: pp. 291302. 
[52] B. Arres, N. Kabbachi, O. Boussaid, Building OLAP cubes on a Cloud Computing environment with MapReduce, in: Comput. Syst. Appl. AICCSA 2013 ACS Int. Conf. On, IEEE, 2013: pp. 1-5.

[53] B. Palanisamy, A. Singh, L. Liu, \& B. Langston, Cura: A Cost-optimized Model for MapReduce in a cloud, in: 'IPDPS', IEEE Computer Society, (2013), pp. 1275-1286.

[54] Y. Wang, A. Song, J. Luo, A MapReduceMerge-based Data Cube Construction Method, in: IEEE, 2010: pp. 1-6. [55] S. Kurunji, T. Ge, B. Liu, C.X. Chen, Communication cost optimization for cloud Data Warehouse queries., in: CloudCom, Citeseer, 2012: pp. 512-519. [56] J. Lee, T. Feng, W. Shi, A. Bedagkar-Gala, S.K. Shah, H. Yoshida, Towards Quality Aware Collaborative Video Analytic Cloud, in: IEEE, 2012: pp. 147-154.

[57] A. Abelló, J. Ferrarons, O. Romero, Building cubes with MapReduce, in: Proc. ACM 14th Int. Workshop Data Warehous. OLAP, ACM, 2011: pp. 17-24. [58] Y. Hua, X. Liu, H. Jiang, ANTELOPE: A Semantic-Aware Data Cube Scheme for Cloud Data Center Networks, IEEE Trans. Comput. 63 (2014) 2146-2159. [59] V. Chang, The business intelligence as a service in the cloud, Future Gener. Comput. Syst. 37 (2014) 512-534.

[60] Q. Chen, M. Hsu, H. Zeller, Experience in Continuous analytics as a Service (CaaaS), in: Proc. 14th Int. Conf. Extending Database Technol., ACM, 2011: pp. 509-514. [61] G. Yantao, M. Xiaofeng, S. Yingjie, COLA: A Cloud-Based System for Online Aggregation, in: Data Engineering (ICDE), 29th International Conference on. IEEE, 2013. p. 1368-1371.

[62] P. Brezany, Y. Zhang, I. Janciak, P. Chen, S. Ye, An Elastic OLAP Cloud Platform, in: IEEE, 2011: pp. 356-363.

[63] H. Al-Aqrabi, L. Liu, R. Hill, N. Antonopoulos, Cloud BI: Future of business intelligence in the Cloud, J. Comput. Syst. Sci. 81 (2015) 85-96.

[64] H. Al-Aqrabi, L. Liu, R. Hill, N. Antonopoulos, Taking the Business Intelligence to the Clouds, in: IEEE, 2012: pp. 953-958.

[65] T.W. Wlodarczyk, C. Rong, B. Jia, L. Cocanu, C.I. Nyulas, M.A. Musen, DataStorm An Ontology-Driven Framework for Cloud-Based Data Analytic Systems, in: IEEE, 2010: pp. 123-127.

[66] S. Chaisiri, Z. Bong, C. Lee, B.-S. Lee, P. Sessomboon, T. Saisillapee, et al., Workflow framework to support data analytics in cloud computing, in: Cloud Comput. Technol. Sci. CloudCom 2012 IEEE 4th Int Conf. On, IEEE, 2012: pp. 610-613 [67] S. Soumya, D. Debabrata, C. Nabendu, An Architecture to Maintain Materialized View in Cloud Computing Environment for OLAP Processing, ICCS 2012 Int. Conf. pp.360,365, 14-15 Sept. 2012

[68] I. Patel, A. Rau-Chaplin, B. Varghese, A Platform for Parallel R-based Analytics on Cloud Infrastructure, in: Parallel Process. Workshop ICPPW 2012 41st Int. Conf. On, IEEE, 2012: pp. 188-193.

[69] D. Lian, X. Li Da, Business intelligence for enterprise systems: a surve, 8 (2012) 679-687.

[70] D. Talia, Toward Cloud-Based Big Data Analytics, Computer. 46, 5, p. 98-101. (2013).

[71] C. Goncalves, L. Assuncao, J.C. Cunha, Data analytics in the cloud with flexible MapReduce workflows., in: CloudCom, Citeseer, 2012: pp. 427-434. [72] N. Chohan, A. Gupta, C. Bunch, K. Prakasam, C. Krintz, Hybrid Cloud support for large scale analytics and web processing, in: Proc. 3rd USENIX Conf. Web Appl. Dev., USENIX Association, 2012: pp. 4-4. [73] R.S. Barga, J. Ekanayake, W. Lu, Project Daytona: Data Analytics as a Cloud Service, in: IEEE, 2012: pp. 1317-1320.

[74] R. Mian, P. Martin, J.L. Vazquez-Poletti, Provisioning data analytic workloads in a cloud, Future Gener. Comput. Syst. 29 (2013) 1452-1458.

[75] B. Yuan, J. Herbert, A Cloud-Based Mobile Data Analytics Framework: Case Study of Activity Recognition Using Smartphone, in: Mob. Cloud Comput. Serv. Eng. MobileCloud 2014 2nd IEEE Int.

[76] G. Shanks, R. Sharma, P. Seddon, P. Reynolds, The impact of strategy and maturity on business analytics and firm performance: a review and research agenda, in: Proc 21st Australas. Conf. Inf. Syst., 2010.

[77] S. Leimeister, M. Böhm, C. Riedl, H. Krcmar, The Business Perspective of Cloud Computing: Actors, Roles and Value Networks., in: ECIS, 2010.

[78] A. Gatzioura, A.Menychtas, V.Moulos \& T. Varvarigou, Incorporating Business Intelligence in Cloud Marketplaces, In: Parallel and Distributed Processing with Applications (ISPA), 2012 IEEE 10th International Symposium on. IEEE, 2012. p. 466-472. [79] G. Aceto, A. Botta, W. de Donato, A. Pescapè, Cloud monitoring: A survey, Comput. Netw. 57 (2013) 2093-2115.

[80] H. Demirkan, D. Delen, Leveraging the capabilities of service-oriented decision support systems: Putting analytics and big data in cloud, Decis. Support Syst. 55 (2013) 412-421. [81] M. Kowalczyk, P. Buxmann, J. Besier, Investigating Business Intelligence And Analytics From A Decision Process Perspective: A Structured, (2013).

[82] S. Subashini, V. Kavitha, A survey on security issues in service delivery models of cloud computing, J. Netw. Comput. Appl. 34 (2011) 1-11. 
[83] W.S. Ng, M. Kirchberg, S. Bressan, K.-L. Tan, Towards a privacy-aware stream data management system for cloud applications, Int. J. Web Grid Serv. 7 (2011) 246-267.

[84] M.D. Assunção, R.N. Calheiros, S. Bianchi, M.A.S. Netto, R. Buyya, Big Data computing and clouds: Trends and future directions, J. Parallel Distrib. Comput. (2014).

[85] O. Sangupamba Mwilu, N. Prat, I. Comyn-Wattiau, Taxonomy development for complex emerging technologies - The case of Business Intelligence and Analytics On the cloud, in PACIS: 2015. 